\title{
EXTENSIONS OF ORDERED GROUPS ${ }^{1}$
}

\section{PAUL CONRAD}

1. Introduction and preliminary results. Throughout this paper let $G$ denote an additive linearly ordered group (notation $o$-group). Let $\Gamma$ denote the set of all pairs of convex subgroups $G^{\gamma}, G_{\gamma}$ of $G$ such that $G^{\gamma}$ covers $G_{\gamma}$ (we identify $\gamma$ with the pair $G^{\gamma}, G_{\gamma}$ ). $\Gamma$ is simply ordered by inclusion, and the rank of $G$ is the order type of $\Gamma$. Each $G_{\gamma}$ is normal in $G^{\gamma}$ and $G^{\gamma} / G_{\gamma}$ (called a component of $G$ ) is $o$-isomorphic to a subgroup of the additive group $R$ of real numbers. $G$ is called $d$ closed if $n G=G$ for all $n \in I$ where $I$ is the set of positive integers. $G$ is an a-extension of a subgroup $H$ of $G$ if for every $g>0 \in G$ there exist $h>0 \in H$ and $n \in I$ such that $n h \leqq g \leqq(n+1) h . G$ is $a$-closed if it does not admit any proper $a$-extensions. There exists an $a$-closed $a$-extension $H$ of $G$ (see [2]). If $G$ is abelian, then there exists an $a$-closed $a$-extension $H$ of $G$ such that every component of $H$ is $o$-isomorphic to $R$ and $H$ is $d$-closed. In $\S 4$ we show that this does not hold for nonabelian $o$-groups. In $\S 3$ we prove that if every component of $G$ is $d$-closed, then there exists an $a$-extension of $G$ with every component $o$-isomorphic to $R$.

Every group can be embedded in a $d$-closed group (see [6]). If $G$ is abelian, then there exists a $d$-closed $o$-extension $H$ of $G$ such that if $h \in H$, then $n h \in G$ for some $n \in I$ (hence $H$ is an $a$-extension of $G$ ). Moreover any other $o$-extension of $G$ that satisfies both these conditions is equivalent to $H . H$ is called the $d$-closure of $G$. In $\$ 4$ we exhibit an $o$-group with no $d$-closed $a$-extension. In $\$ 3$ we derive a necessary and sufficient condition on the components of $G$ for $G$ to be $d$-closed, provided the rank of $G$ is well ordered. We also show that any $a$-closed $o$-group of rank 2 is $d$-closed and has both components $o$-isomorphic to $R$. Finally we illustrate methods of constructing $o$ groups, making use of the normal $o$-extension theory stated in $\$ 2$. We make repeated use of the following induced homomorphism theorem.

I.H.T. Suppose that $A, B, C$, and $D$ are $o$-groups; $\delta$ is an $o$-homomorphism of $A$ onto $D ; \beta$ is an $o$-homomorphism of $B$ into $C$; and $\alpha$ is a (single-valued) mapping of $A$ into $B$ such that $K(\delta) \alpha \leqq K(\beta)$ (where $K(\delta)$ is the kernel of $\delta$ ) and $\alpha \beta$ is an $o$-homomorphism of $A$ into $C$. Then there exists a unique $o$-homomorphism $\alpha^{*}$ of $D$ into $C$ such that $\delta \alpha^{*}=\alpha \beta$. Moreover $\alpha^{*}$ is an isomorphism if and only if $K(\delta)$

Presented to the Society, November 27, 1954; received by the editors September 27, 1954.

1 This work was done under contract N7-onr-434, Task Order III, Navy Department, The Office of Naval Research, U.S.A. 
$=\alpha^{-1} K(\beta)$. The proof is almost identical with the proof of the corresponding theorem for nonordered groups.

For any $g \neq 0 \in G$ there exists a unique $\gamma \in \Gamma$ (called the value of $g$ and denoted by $V(g))$ such that $g \in G^{\gamma} \backslash G_{\gamma}$. We define $V(0)<\gamma$ for all $\gamma \in \Gamma$ where 0 is the identity of $G$. We made use of the fact that if $\pi$ is an $o$-automorphism of a subgroup $K$ of $R$, then $k \pi=p k$ for all $k \in K$ where $p$ is a fixed element of the set $P$ of all positive real numbers.

(Added in proof. The referee has pointed out that the method of proof of Theorem 3.1 was used by P. Jafford [4]).

2. Normal o-extensions of o-groups. The extension theory for groups that was developed by $O$. Schreier [7] applies with slight modifications to $o$-groups. This theory has been applied to partially ordered groups by L. Fuchs [3]. F. W. Levi [5] effectively points out that a normal extension $G$ of an $o$-group $N$ by another 0 -group $N^{\prime}$ can be ordered so as to induce the given orders in $N$ and $N^{\prime}$ if and only if the inner automorphisms of $G$ induce $o$-automorphisms of $N$. The proofs of the statements in this section will be omitted, since they are almost identical with the classical ones. Let $N$ and $N^{\prime}$ be $o$-groups with elements $a, b, c, \cdots$ and $a^{\prime}, b^{\prime}, c^{\prime}, \cdots$ and identities 0 and $0^{\prime}$ respectively. Suppose that $G$ is a normal o-extension of $N$ by $N^{\prime}$. That is, there exists an $o$-homomorphism $\pi$ of $G$ onto $N^{\prime}$ with kernel $N$. Then $N$ is a convex subgroup of $G$. A mapping $r$ of $N^{\prime}$ into $G$ such that $r\left(0^{\prime}\right)=0$ and $r\left(a^{\prime}\right) \pi=a^{\prime}$ is called a representative function. The factor set derived from $r$ is the mapping $f$ of $N^{\prime} \times N^{\prime}$ into $N$ defined by: $f\left(a^{\prime}, b^{\prime}\right)=-r\left(a^{\prime}+b^{\prime}\right)+r\left(a^{\prime}\right)+r\left(b^{\prime}\right)$. For $x$ and $y$ in $G$ we define $x y=-y+x+y$. Then $(x y) z=x(y+z)$, and we have

(1) $\left[\operatorname{ar}\left(a^{\prime}\right)\right] r\left(b^{\prime}\right)=\left[\operatorname{ar}\left(a^{\prime}+b^{\prime}\right)\right] f\left(a^{\prime}, b^{\prime}\right)$, and $\operatorname{ar}\left(0^{\prime}\right)=a$,

(2) $r\left(a^{\prime}\right)+a+r\left(b^{\prime}\right)+b=r\left(a^{\prime}+b^{\prime}\right)+f\left(a^{\prime}, b^{\prime}\right)+a r\left(b^{\prime}\right)+b$,

(3) $f\left(a^{\prime}, 0^{\prime}\right)=f\left(0^{\prime}, b^{\prime}\right)=0$, and

(4) $f\left(a^{\prime}, b^{\prime}+c^{\prime}\right)+f\left(b^{\prime}, c^{\prime}\right)=f\left(a^{\prime}+b^{\prime}, c^{\prime}\right)+f\left(a^{\prime}, b^{\prime}\right) r\left(c^{\prime}\right)$.

Conversely suppose that $f$ is a mapping of $N^{\prime} \times N^{\prime}$ into $N$, and $r$ is a mapping of $N^{\prime}$ into the group of $o$-automorphisms of $N$. Suppose further that (1), (3), and (4) are satisfied. Let $\bar{G}=N^{\prime} \times N$, and define $\left(a^{\prime}, a\right)+\left(b^{\prime}, b\right)=\left(a^{\prime}+b^{\prime}, f\left(a^{\prime}, b^{\prime}\right)+a r\left(b^{\prime}\right)+b\right)$. Next define $\left(a^{\prime}, a\right)$ positive if $a^{\prime}>0^{\prime}$ or $a^{\prime}=0^{\prime}$ and $a>0$. Then $\bar{G}$ is a normal $o$-extension of $\bar{N}=0^{\prime} \times N$ by $N^{\prime}$. Thus if $G$ is a normal $o$-extension of $N$ by $N^{\prime}$, then by using a representative function $r\left(a^{\prime}\right)$ and the derived factor set $f\left(a^{\prime}, b^{\prime}\right)$ we can construct $\bar{G}$. The mapping of $r\left(a^{\prime}\right)+a$ onto $\left(a^{\prime}, a\right)$ is an $o$-isomorphism of $G$ onto $\bar{G}$. We shall frequently make use of this representation of $G$. We next consider some special cases of the above theory. 
I. Suppose that $N$ is abelian. Then the first part of (1) reduces to $\left[\operatorname{ar}\left(a^{\prime}\right)\right] r\left(b^{\prime}\right)=\operatorname{ar}\left(a^{\prime}+b^{\prime}\right)$. Hence $r$ is a homomorphism of $N^{\prime}$ into the group of all $o$-automorphisms of $N$.

II. Suppose that $G$ splits over $N$. Then we can (and always shall) pick a representative function $r$ so that $r\left(a^{\prime}+b^{\prime}\right)=r\left(a^{\prime}\right)+r\left(b^{\prime}\right)$, and hence $f\left(a^{\prime}, b^{\prime}\right) \equiv 0$. Thus $G$ is determined by $N, N^{\prime}$ and a homomorphism of $N^{\prime}$ into the group of $o$-automorphisms of $N$.

III. Suppose that $N$ is in the center of $G$. Then $\operatorname{ar}\left(a^{\prime}\right)=a^{\prime}$ for all $a$ and $a^{\prime}$. Hence $G$ is completely determined by $N, N^{\prime}$ and a mapping $f$ of $N^{\prime} \times N^{\prime}$ into $N$ that satisfies (3) and $f\left(a^{\prime}, b^{\prime}+c^{\prime}\right)+f\left(b^{\prime}, c^{\prime}\right)$ $=f\left(a^{\prime}+b^{\prime}, c^{\prime}\right)+f\left(a^{\prime}, b^{\prime}\right)$.

Let $\left(G_{1}, \pi_{1}\right)$ and $\left(G_{2}, \pi_{2}\right)$ be normal $o$-extensions of $N$ by $N^{\prime}$. They are called equivalent extensions if there exists an $o$-isomorphism $\sigma$ of $G_{1}$ on to $G_{2}$ such that $a \sigma=a$ for all $a \in N$. It follows by the I.H.T. that there exists an $o$-automorphism $\tau$ of $N^{\prime}$ such that $\sigma \pi_{2}=\pi_{1} \tau$. If $\pi$ is an $o$-isomorphism of $G_{1}$ onto $G_{2}$, then (since $N$ is convex) $N \pi \leqq N$ or $N \pi>N$. If the rank of $G_{1}$ is well ordered, then $N \pi=N$. Hence in this case the two extensions are equivalent if and only if the $o$-automorphism of $N$ that is induced by $\pi$ can be extended to an $o$-automorphism of $G_{1}$. In general the two extensions are equivalent if and only if there exist an $o$-automorphism $\tau$ of $N^{\prime}$ and a mapping $g$ of $N^{\prime}$ into the center of $N$ such that $a r_{1}\left(a^{\prime}\right)=a r_{2}\left(a^{\prime} \tau\right)$ and $f_{2}\left(a^{\prime} \tau, b^{\prime} \tau\right)-f_{1}\left(a^{\prime}, b^{\prime}\right)$ $=g\left(a^{\prime}+b^{\prime}\right)-g\left(a^{\prime}\right)-g\left(b^{\prime}\right)$.

3. Some extension theorems. Let $S$ be the set of all abelian convex subgroups $A$ of $G$. Let $M=\cup_{A \in S} A$. Then $M$ is the greatest abelian convex subgroup of $G$. Clearly $M$ is mapped onto itself by every $o$ automorphism of $G$.

THEOREM 3.1. There exists an a-extension $H$ of $G$ such that: (i) $H$ contains a d-closure $D$ of $M$; (ii) $H$ is generated by $D$ and $G$; and (iii) if $K$ is an a-extension of $G$ that satisfies (i) and (ii), then $K$ is equivalent to $H$.

We first prove the following

Lemma. Suppose that $M$ is an abelian o-group, $D$ is a d-closure of $M$, and $\pi$ is an o-automorphism of $M$. Then there exists one and only one o-automorphism $\sigma$ of $D$ such that $a \sigma=a \pi$ for all $a \in M$.

Proof. For $d \in D$ we define $d \sigma=(1 / m)(m d \pi)$ where $m \in I$ and $m d \in M$ (i.e., $d \sigma$ is the element $x \in D$ such that $m x=m d \pi$ ). If $m d$ and $n d$ are in $M$, then $m^{2} n^{2}[(1 / m)(m d \pi)]=m n[n(m d \pi)]=m n[m(n d \pi)]$ $=m^{2} n^{2}[(1 / n)(n d \pi)]$. Hence $(1 / n)(n d \pi)=(1 / m)(m d \pi)$, and $\sigma$ is a (single-valued) mapping of $D$ into itself. It is easy to show that $\sigma$ is 
an 0 -automorphism of $D$ such that $a \sigma=a \pi$ for all $a \in M$. Suppose that $\rho$ is an $o$-automorphism of $D$ such that $a \rho=a \pi$ for all $a \in M$. Consider any $d \in D$. Then $n d \in M$ for some $m \in I . n(d \rho)=(n d) \rho=(n d) \pi=(n d) \sigma$ $=n(d \sigma)$. Therefore $d \sigma=d \rho$.

Proof of THE THEOREM. $G$ is a normal $o$-extension of $M$ by $G / M$. Hence there exist a representative function $r\left(a^{\prime}\right)$ and a derived factor set $f\left(a^{\prime}, b^{\prime}\right)$ of this extension. We use the representation $\bar{G}$ of $G$. Let $D$ be a $d$-closure of $M$. For every $a^{\prime}$ in $G / M$, let $s\left(a^{\prime}\right)$ be the unique extension of the $o$-automorphism $r\left(a^{\prime}\right)$ of $M$ to an $o$-automorphism of $D$. Next consider any $d \in D$. There exists an $m \in I$ such that $m d \in M$. $m\left[d s\left(a^{\prime}+b^{\prime}\right)\right]=(m d) s\left(a^{\prime}+b^{\prime}\right)=(m d) r\left(a^{\prime}+b^{\prime}\right)=\left[m d r\left(a^{\prime}\right)\right] r\left(b^{\prime}\right)$ $=\left[m d s\left(a^{\prime}\right)\right] s\left(b^{\prime}\right)=m\left[d s\left(a^{\prime}\right)\right] s\left(b^{\prime}\right)$. Thus $s\left(a^{\prime}+b^{\prime}\right)=s\left(a^{\prime}\right) s\left(b^{\prime}\right)$. Hence $s$ is a homomorphism of $G / M$ into the group of $o$-automorphisms of $D$. Let $H=G / M \times D$, and define $\left(a^{\prime}, a\right)+\left(b^{\prime}, b\right)=\left(a^{\prime}+b^{\prime}, f\left(a^{\prime}, b^{\prime}\right)\right.$ $\left.+a s\left(a^{\prime}\right)+b\right)$. As usual define $\left(a^{\prime}, a\right)$ positive if $a^{\prime}>0^{\prime}$ or $a^{\prime}=0^{\prime}$ and $a>0$. One easily verifies that $H$ is an $a$-extension of $\bar{G}$ that satisfies (i) and (ii).

Now suppose that $H$ and $H^{\prime}$ are $a$-extensions of $G$ that satisfy (i) and (ii). Let $D$ and $D^{\prime}$ be the $d$-closures of $M$ in $H$ and $H^{\prime}$ respectively. Since $H\left(H^{\prime}\right)$ is generated by $D$ and $G\left(D^{\prime}\right.$ and $\left.G\right)$, the set of representatives $r\left(a^{\prime}\right)$ of $G / M$ is also a set of representatives of $H / D$ $\left(H^{\prime} / D^{\prime}\right) . D$ and $D^{\prime}$ are equivalent extensions of $M$. Therefore there exists an $o$-isomorphism $\pi$ of $D$ onto $D^{\prime}$ such that $a \pi=a$ for all $a \in M$. For any $d \in D$ there exists an $n \in I$ such that $n d \in M . n\left(d \pi r\left(a^{\prime}\right)\right)$ $=(n d \pi) r\left(a^{\prime}\right)=n d r\left(a^{\prime}\right)=n d r\left(a^{\prime}\right) \pi=n\left(d r\left(a^{\prime}\right) \pi\right)$. Hence $\pi r\left(a^{\prime}\right)=r\left(a^{\prime}\right) \pi$ for all $a^{\prime}$.

Every $h \in H$ has a unique representation $h=r\left(a^{\prime}\right)+d$ where $d \in D$. Consider the mapping $\sigma: h \sigma=\left(r\left(a^{\prime}\right)+d\right) \sigma=r\left(a^{\prime}\right)+d \pi$ of $H$ into $H^{\prime}$. $\left(r\left(a^{\prime}\right)+a+r\left(b^{\prime}\right)+b\right) \sigma=\left(r\left(a^{\prime}+b^{\prime}\right)+f\left(a^{\prime}, b^{\prime}\right)+a r\left(b^{\prime}\right)+b\right) \sigma=r\left(a^{\prime}+b^{\prime}\right)$ $+f\left(a^{\prime}, \quad b^{\prime}\right) \pi+a r\left(b^{\prime}\right) \pi+b \pi=r\left(a^{\prime}+b^{\prime}\right)+f\left(a^{\prime}, b^{\prime}\right)+a \pi r\left(b^{\prime}\right)+b \pi=r\left(a^{\prime}\right)$ $+a \pi+r\left(b^{\prime}\right)+b \pi=\left(r\left(a^{\prime}\right)+a\right) \sigma+\left(r\left(b^{\prime}\right)+b\right) \sigma$. Thus $\sigma$ is a homomorphism, and it is easy to verify that $\sigma$ is an $o$-isomorphism of $H$ onto $H^{\prime}$ such that $g \sigma=g$ for all $g \in G$.

Theorem 3.2. Suppose that $G$ has a $d$-closed component, say $G^{\beta} / G_{\beta}$. Then there exists an a-extension $H$ of $G$ for which $H^{r} / H_{r} \cong R$ whenever there exists an inner automorphism of $G$ that maps $G^{\beta}$ onto $G^{\gamma}$, otherwise $H^{\gamma} / H_{\gamma} \cong G^{\gamma} / G_{\gamma}$.

Corollary. Suppose that all of the components of $G$ are $d$-closed. Then there exists an a-closed a-extension of $G$ with each component o-isomorphic to $R$.

The corollary follows at once from the theorem by the usual trans- 
finite arguments. To prove the theorem we need the following definitions, lemmas, and diagram. For $g \in G, \pi(g)$ will denote the inner automorphism of $G$ determined by $g$. That is $x \pi(g)=-g+x+g$ for all $x \in G$. $\pi$ is a homomorphism of $G$ into the group of all $o$-automorphisms of $G$.

For $h, k \in G$ we define $V(k) \tau(h)=V(k \pi(h))$.

Lemma. $\tau$ is a homomorphism of $G$ into the group of all the order preserving permutations of $\Gamma$.

Proof. We first show that for every $h \neq 0, k \neq 0, g \in G, V(h)=V(k)$ if and only if $V(h \pi(g))=V(k \pi(g))$. For if $V(h)=V(k)=\gamma$, then $h, k \in G^{\gamma} \backslash G_{\gamma}$. Hence $h \pi(g), \quad k \pi(g) \in G^{\gamma} \pi(g) \backslash G_{\gamma} \pi(g)$. But $G^{\gamma} \pi(g)$, $G_{\gamma} \pi(g) \in \Gamma$ (since $\pi(g)$ is an $o$-automorphism). Thus $V(h \pi(g))$ $=V(k \pi(g))$. Conversely if $V(h \pi(g))=V(k \pi(g))$, then $V(h)=V(h \pi(g)$ $\cdot \pi(-g))=V(k \pi(g) \pi(-g))=V(k)$. Therefore $\tau$ is a (single-valued) mapping of $G$ into the set of all 1-1 mappings of $\Gamma$ into itself. Since $\pi(h)$ is a mapping of $G$ onto $G$ and $V$ is a mapping of $G$ onto $\Gamma$, each $\tau(h)$ is a mapping of $\Gamma$ onto itself. If $V(h)<V(k)$, then $V(h \pi(g)) \neq V(k \pi(g))$. Without loss of generality $0<h<k$. Then $0<h \pi(g)<k \pi(g)$, hence $V(h \pi(g))<V(k \pi(g))$. Therefore each $V(h)$ is an order preserving permutation of $\Gamma$. Finally $\tau(k) \tau(g+h)=V(k \pi(g+h))=V([k \pi(g)]$ $\cdot \pi(h))=V[k \pi(g)] \tau(h)=[V(k) \tau(g)] \tau(h)$.

For $\beta \in \Gamma$ we define $\Delta_{\beta}=\left\{\delta \in \Gamma \mid G^{\beta} \pi(g)=G^{\delta}\right.$ for some $\left.g \in G\right\}$.

Lemma. $\Delta_{\beta}=\{\delta \in \Gamma \mid V(k)=\beta$ and $V(k \pi(g))=\delta$ for some $h, k \neq 0 \in G\}$ $=\{\delta \in \Gamma \mid \beta \tau(g)=\delta$ for some $g \in G\} . G^{\delta} / G_{\delta}$ is o-isomorphic to $G^{\beta} / G_{\beta}$ for every $\delta \in \Delta_{\beta}$.

The equalities follow from the last lemma and the fact that each $\tau(h)$ induces an $o$-permutation of $\Delta_{\beta}$. The last part of the lemma is an immediate consequence of the I.H.T.

Now $G^{\beta} / G_{\beta}$ is $o$-isomorphic to a subgroup $R_{\beta}$ of $R$. Hence for each $\delta \in \Delta_{\beta}$ there exists an $o$-isomorphism $\pi_{\delta}$ of $G^{\delta} / G_{\delta}$ onto $R_{\beta}$. The set $\left\{\pi_{\delta} \mid \delta \in \Delta_{\beta}\right\}$ will be kept fixed throughout the following discussion. Then for each $\delta \in \Delta_{\beta}$ we have the diagram on the following page, where the $\sigma_{\delta}$ are the natural o-homomorphisms. $K\left(\sigma_{\delta} \pi_{\delta}\right) \pi(h)$ $=G_{\delta} \pi(h)=G_{\delta r(h)}=K\left(\sigma_{\delta \tau(h)} \pi_{\delta \tau(h)}\right)$. Therefore by the I.H.T., $\pi(h)$ induces an $o$-automorphism $p(\delta, h)$ of $R_{\beta}$ such that $\sigma_{\delta} \pi_{\delta} p(\delta, h)$ $=\pi(h) \sigma_{\delta \tau(h)} \pi_{\delta \tau(h)}$. Similarly $\pi(k)$ induces an $o$-automorphism $p(\delta \tau(h), k)$ of $R_{\beta}$ such that $\sigma_{\delta \tau(h)} \pi_{\delta \tau(h)} p(\delta \tau(h), k)=\pi(k) \sigma_{\delta \tau(h+k)} \pi_{\delta \tau(h+k)}$, and $\pi(h+k)$ induces an $o$-automorphism $p(\delta, h+k)$ of $R_{\beta}$ such that $\sigma_{\delta} \pi_{\delta} p(\delta, h+k)$ $=\pi(h+k) \sigma_{\delta \tau(h+k)} \pi_{\delta \tau(h+k)}$. But $\sigma_{\delta} \pi_{\delta} p(\delta, h+k)=\pi(h) \pi(k) \sigma_{\delta \tau(h+k)} \pi_{\delta \tau(h+k)}$ $=\pi(h) \sigma_{\delta \tau(h)} \pi_{\delta \tau(h)} p(\delta \tau(h), k)=\sigma_{\delta} \pi_{\delta} p(\delta, h) p(\delta \tau(h), k)$. Therefore $p(\delta, h)$ 


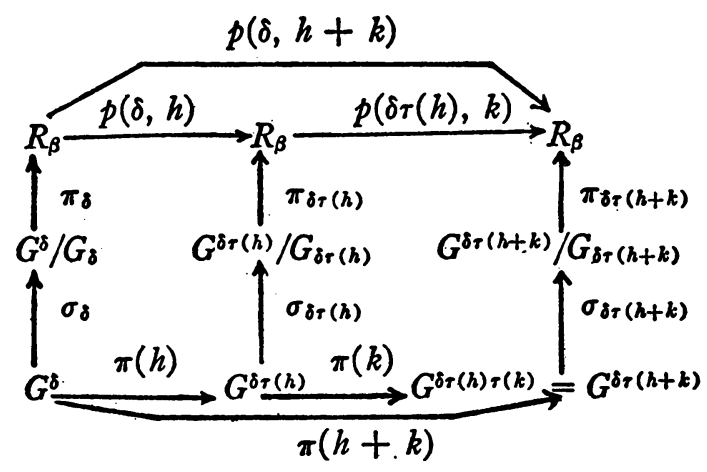

- $p(\delta \tau(h), k)=p(\delta, h+k)$. Finally without loss of generality $p(\delta, h) \in P$ for all $\delta \in \Delta_{\beta}$ and $h \in G$.

Proof of theorem. Since $G^{\beta} / G_{\beta}$ is $d$-closed, $R=R_{\beta} \oplus K_{\beta}$. Form the small direct sum $K$ of the $K_{\delta}$ where $K_{\delta}=K_{\beta}$ for all $\delta \in \Delta_{\beta}$. Denote the identity of $K$ by $\phi$. Define $k=\left(\cdots, k_{\delta}, \cdots\right) \in K$ positive if $k \neq \phi$ and the nonzero component with greatest $\delta$ is positive. Then $K$ is an abelian $o$-group that we wish to adjoin to $G$.

For $k \in K$ and $g \in G$ we define $k p(g)=\left(\cdots,\left[k_{\delta} p(\delta, g)\right]_{\delta \tau(o)}, \cdots\right)$. $(h+k) p(g)=\left(\cdots,\left[(h+k)_{\delta} p(\delta, g)\right]_{\delta \dot{\gamma}(g)}, \cdots\right)=\left(\cdots,\left[\left(h_{\delta}+k_{\delta}\right) p(\delta\right.\right.$, g) $\left.]_{\delta \tau(g)}, \cdots\right)=\left(\cdots,\left[h_{\delta} p(\delta, g)\right]_{\delta \tau(g)}, \cdots\right)+\left(\cdots,\left[k_{\delta} p(\delta, g)\right]_{\delta \tau(\theta)}\right.$, $\cdots)=h p(g)+k p(g)$. If $k p(g)=\phi$, then $k_{\delta} p(\delta, g)=0$ for all $\delta \in \Delta_{\beta}$. Hence $k_{\delta}=0$ for all $\delta$, and $k=\phi \cdot x p(g)=k$ has a solution $x$ for every $k \in K$. Simply let $x_{\delta}=k_{\delta \tau(g)} / p(\delta, g)$. Thus we have shown that $p(g)$ is an automorphism of $K \cdot k p(g+h)=\left(\cdots,\left[k_{\delta} p(\delta, g+h)\right]_{\delta \tau(o+h)}, \cdots\right)$ $=\left(\cdots,\left[k_{\delta} p(\delta, g) p(\delta \tau(g), h)\right]_{\delta \tau(\theta) \tau(h)}, \cdots\right)=\left(\cdots,\left[k_{\delta} p(\delta, g)\right]_{\delta \tau(\theta)}\right.$, $\cdots) p(h)=[k p(g)] p(h)$. Therefore $p$ is a homomorphism of $G$ into the group of automorphisms of $K$.

Let $H=G \times K$, and define $\left(g_{1}, k_{1}\right)+\left(g_{2}, k_{2}\right)=\left(g_{1}+g_{2}, k_{1} p\left(g_{2}\right)+k_{2}\right)$. Then $H$ is a splitting extension of $K$ by $G$. Define $(g, k) \in H$ positive if $V(g)>V(k)$ and $g>0$, or $V(k)>V(g)$ and $k>\phi$, or $V(g)=V(k)=\delta$ and $g \sigma_{\delta} \pi_{\delta}+k_{\delta}>0$. It follows by a long straightforward computation that $H$ is an $o$-group. In fact $H$ is an $a$-extension of $\{(g, \phi) \mid g \in G\}$. Finally if $\delta \in \Delta_{\beta}$, then the mapping of $(g, k) \in H^{\delta}$ onto $g \sigma_{\delta} \pi_{\delta}+k_{\delta}$ is an $o$-homomorphism of $H^{\delta}$ onto $R$ with kernel $H_{\delta}$, and if $\gamma \in \Gamma \backslash \Delta_{\beta}$, then the mapping of $(g, k) \in H^{\gamma}$ onto $g+G_{\gamma}$ is an $o$-homomorphism of $H^{\gamma}$ onto $G^{\gamma} / G_{\gamma}$ with kernel $H_{\gamma}$.

Assume that $G$ has a convex subgroup $G^{1}$ that covers 0 . Then by Theorem 3.1 there exists an $a$-extension $K$ of $G$ such that $K^{1}$ is $d$ closed. Hence by Theorem 3.2 there exists an $a$-extension $H$ of $G$ such that $H^{1} \cong R$. This result can be sharpened. 
Theorem 3.3. If $G$ has a convex subgroup $G^{1}$ that covers 0 , then there exists an a-extension $H$ of $G$ such that (i) $H^{1} \cong R$, (ii) $H$ is generated by $H^{1}$ and $G$, and (iii) if $K$ is an a-extension of $G$ that satisfies (i) and (ii), then $K$ is equivalent to $H$.

The proof is entirely similar to the proof of Theorem 3.1. For if $\pi$ is an $o$-automorphism of a subgroup $G^{1}$ of $R$, then $a \pi=p a$ for all $a \in G^{1}$ where $p$ is a fixed element of $P$. Hence there exists a unique extension of $\pi$ to an $o$-automorphism of $R$.

Suppose that the inner automorphism $\pi(g)$ of $G$ maps $G^{\gamma}$ onto itself, then we have the following diagram:

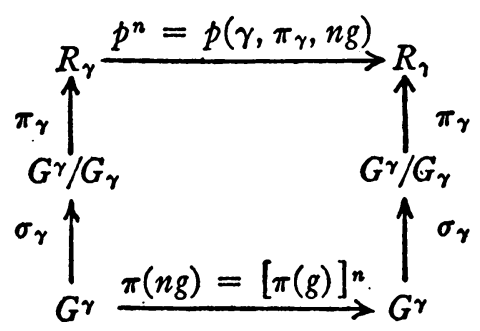

where $p$ is an $o$-automorphism of $R_{\gamma}$, hence without loss of generality $p \in P$.

LemMA. If $G$ is $d$-closed, then $1+p+\cdots+p^{n}$ is an o-automorphism of $R_{\gamma}$ for every $n \in I$.

Proof. It is sufficient to show that $\left(1+p+\cdots+p^{n}\right) R_{\gamma}=R_{\gamma}$. Clearly $\left(1+p+\cdots+p^{n}\right) R_{\gamma} \leqq R_{\gamma}$. Since $G$ is $d$-closed, $R_{\gamma}$ is $d$-closed. If $V(g) \leqq \gamma$, then $p=1$. Hence $\left(1+p+\cdots+p^{n}\right) R_{\gamma}=n R_{\gamma}=R_{\gamma}$. Next assume that $V(g)>\gamma$. Consider any $h \in G$ with value $\gamma$ and any $n \in I$. Then there exists an element $d \in G$ such that $(n+1) d=(n+1) g+h$ $\equiv(n+1) g \bmod G^{\gamma}$. Hence $d=g+x$ where $V(x)=\gamma . \quad(n+1) g+h$ $=(n+1)(g+x)=(n+1) g+x \pi[n g]+x \pi[(n-1) g]+\cdots+x \pi[g]+x$. Therefore $h \sigma_{\gamma} \pi_{\gamma}=x \pi[n g] \sigma_{\gamma} \pi_{\gamma}+\cdots+x \pi[g] \sigma_{\gamma} \pi_{\gamma}+x \sigma_{\gamma} \pi_{\gamma}=p^{n}\left[x \sigma_{\gamma} \pi_{\gamma}\right]$ $+\cdots+p\left[x \sigma_{\gamma} \pi_{\gamma}\right]+x \sigma_{\gamma} \pi_{\gamma}=\left(p^{n}+\cdots+p+1\right)\left[x \sigma_{\gamma} \pi_{\gamma}\right]$. This completes the proof, since $h \sigma_{\gamma} \pi_{\gamma}$ is an arbitrary element of $R_{\gamma}$.

THEOREM 3.4. If the rank of $G$ is well ordered, then the following statements are equivalent.

(A) $G$ is $d$-closed.

(B) If $p$ is an o-automorphism of $R_{\gamma}$ that is induced by an inner automorphism of $G$, then $1+p+\cdots+p^{n}$ is an o-automorphism of $R_{\gamma}$ (for every $\gamma \in \Gamma$ and $n \in I)$.

Proof. By the last lemma (A) implies (B). Now assume that (B) is true. Since $\Gamma$ is well ordered, every $G^{\boldsymbol{\gamma}}$ is normal. Hence for every 
$\gamma \in \Gamma$ and $g \in G, \pi(g)$ induces an $o$-automorphism $p\left(\gamma, \pi_{\gamma}, g\right)$ of $R_{\gamma}$. In particular $1=p\left(\gamma, \pi_{\gamma}, 0\right)$ for every $\gamma \in \Gamma$. Therefore $n R_{\gamma}=\left(1+1^{2}\right.$ $\left.+\cdots+1^{n}\right) R_{\gamma}=R_{\gamma}$. Therefore every component of $G$ is $d$-closed.

Next consider any $g \neq 0$ in $G$ and any $n \in I$. Let $V(g)=\alpha$. Then since $G^{\alpha} / G_{\alpha}$ is $d$-closed, there exists $X=G_{\alpha}+a$ in $G^{\alpha} / G_{\alpha}$ such that $n X=G_{\alpha}+g$. Hence $n a \equiv g \bmod G_{\alpha}$. Let $\Pi=\{\gamma \in \Gamma \mid$ there exists $h_{\gamma} \in G$ such that $\left.n h_{\gamma} \equiv g \bmod G_{\gamma}\right\}$. We note that $\Pi$ contains all $\gamma \in \Gamma$ such that $\gamma \geqq \alpha$. Thus $I$ contains a least element, say $\beta$. Since $0=G_{1}<G^{1}=G_{2}<G^{2}=G_{3}<\cdots$, it is sufficient to prove that $\beta=1$. Suppose that $\beta>1$. Then $n h_{\beta}+q=g$ where $q \neq 0$ and $V(q)=\gamma<\beta$. We now show that there exists $y \in G$ such that $V(y)=\gamma$ and $n\left(h_{\beta}+y\right)$ $\equiv g \bmod G_{\gamma}$. But this contradicts our definition of $\beta$. Let $p=p\left(\gamma, \pi_{\gamma}\right.$, $h_{\beta}$ ) and consider any $y \in G^{\gamma} \backslash G_{\gamma}$. Then for any $k \in I$ we have $-k h_{\beta}+y$ $+k h_{\beta}+G_{\gamma}=y \pi\left(k h_{\beta}\right) \sigma_{\gamma}=\left[p^{k}\left(y \sigma_{\gamma} \pi_{\gamma}\right)\right] \pi_{\gamma}^{-1}$. Therefore $n\left(h_{\beta}+y\right)+G_{\gamma}$ $=n h_{\beta}+y \pi\left[(n-1) h_{\beta}\right]+\cdots+y \pi\left[h_{\beta}\right]+y+G_{\gamma}=n h_{\beta}+\left[p^{n-1}\left(y \sigma_{\gamma} \pi_{\gamma}\right)\right]$ $\cdot \pi_{\gamma}^{-1}+\cdots+\left[p\left(y \sigma_{\gamma} \pi_{\gamma}\right)\right] \pi_{\gamma}^{-1}+y \sigma_{\gamma} \pi_{\gamma} \pi_{\gamma}^{-1}=n h_{\beta}+\left[\left(p^{n-1}+\cdots+p+1\right)\right.$ $\left.\cdot\left(y \sigma_{\gamma} \pi_{\gamma}\right)\right] \pi_{\gamma}^{-1}$.

(1) $\left[\left(p^{n-1}+\cdots+p+1\right)\left(y \sigma_{\gamma} \pi_{\gamma}\right)\right] \pi_{\gamma}^{-1}=q \sigma_{\gamma}$ if and only if

(2) $y \sigma_{\gamma} \pi_{\gamma}=q \sigma_{\gamma} \pi_{\gamma} /\left(p^{n-1}+\cdots+p+1\right)$.

Since $\left(p^{n-1}+\cdots+p+1\right) R_{\gamma}=R_{\gamma}$, it follows that the right-hand side of (2) is an element of $R_{\gamma}$. Therefore we can pick $y \in G$ that satisfies (2) and hence (1). But for this $y: n\left(h_{\beta}+y\right)+G_{\gamma}=n h_{\beta}+q \sigma_{\gamma}=n h_{\beta}+q$ $+G_{\gamma}$. Therefore $g=n h_{\beta}+q \equiv n\left(h_{\beta}+y\right) \bmod G_{\gamma}$.

Note that Example 4.1 shows that the well ordering cannot be disregarded. In the following corollaries we assume that the rank of $G$ is well ordered.

I. If $G$ is abelian, then $G$ is $d$-closed if and only if all of its components are $d$-closed.

II. If every component of $G$ is $o$-isomorphic to $R$, then $G$ is $d$-closed.

III. If every component of $G$ is $d$-closed, then there exists an $a$ closed $a$-extension $H$ of $G$ such that $H^{\gamma} / H_{\gamma} \cong R$ for all $\gamma \in \Gamma$, and $H$ is $d$-closed.

THEOREM 3.5. Any a-closed o-group of rank 2 is d-closed and has both components o-isomorphic to $R$.

Proof. Let $G$ be $a$-closed and of rank $2\left(G=G^{2}>G_{2}=G^{1}>G_{1}=0\right)$. Then without loss of generality (by Theorem 3.3) $G^{1}=R$. Consider any pair of elements $g, h \in G \backslash R$ such that $n h=g$ for some $n \in I$. For every $c \in R, c \pi(g)=L c$ and $c \pi(h)=K c$ where $L, K \in P$. But $L c=c \pi(g)$ $=c \pi(n h)=c[\pi(h)]^{n}=K^{n} c$. Therefore $c \pi(h)=L^{1 / n} c$. For any $k \in G \backslash R$, $g \pi(-k)=g+p$ and $h \pi(-k)=h+q$ where $q, p \in R$. We next show that $q$ is completely determined by $n, g$, and $k$. This and the fact that $c \pi(h)$ is determined by $n, g$, and $c$ form the basis of our proof. 
For any $q \in R$ and $m \in I$ we have $m(h+q)=m h+q \pi[(m-1) h]$ $+\cdots+q \pi[h]+q=m h+\left[L^{(m-1) / n}+\cdots+L^{1 / n}+1\right] q$. Let

$$
q=p /\left[L^{(n-1) / n}+\cdots+L^{1 / n}+1\right] .
$$

Then $n(h+q)=n h+p=g+p=k+g-k=k+n h-k=n(k+h-k)$. Therefore $k+h-k=h+q$.

Now assume (by way of contradiction) that $G$ is not $d$-closed. Then there exist $g \in G \backslash R$ and a positive prime $n$ such that $n x=g$ has no solution in $G$. As before $c \pi(g)=L c$ for $c \in R$ and $g \pi(-k)=g+p$ for $k \in G \backslash R$ where $L=L(g) \in P$ and $p=p(g, k) \in R$. Let $q(k)=q(\dot{n}, g, k)$ $=p /\left[L^{(n-1) / n}+\cdots+L^{1 / n}+1\right]$. Let $N$ be the additive group of integers modulo $n$ (represent the elements of $N$ by $0,1,2, \cdots, n-1$ ). For $u, v \in N$ and $h \in G$ we define:

$$
\begin{aligned}
f(u, v) & =\left\{\begin{array}{l}
g \text { if } u+v \geqq n, \\
0 \text { if } u+v<n,
\end{array}\right. \\
h r(u) & =\left\{\begin{array}{l}
L^{u / n} h \text { if } h \in R \text { or } u=0, \\
{\left[L^{(u-1) / n}+\cdots+L^{1 / n}+1\right] q(h)+h \text { if } h \in G / R \text { and } u \neq 0 .}
\end{array}\right.
\end{aligned}
$$

Finally let $K=N \times G$ and define $(u, h)+(v, k)=([u+v] \pi, f(u, v)$ $+h r(v)+k)$ for all $u, v \in N$ and $h, k \in G$ where $\pi$ is the natural homomorphism of the integers onto $N$. To complete this proof it is sufficient to show: (a) $K$ is a group; (b) the mapping of $h \in G$ onto $(0, h)$ is an isomorphism of $G$ on to a subgroup $G^{*}$ of $K$; (c) $K$ can be ordered so that it is a proper $a$-extension of $G^{*}$. For since $G^{*}$ is $a$-closed, it follows that any $a$-closed $o$-group of rank 2 is $d$-closed. Then the latter part of this theorem is an immediate consequence of Theorem 3.2 .

To prove (a) it is sufficient to show that $r(m)$ is an $o$-automorphism of $G$ (for every $m \in N$ ), and that $r$ and $f$ satisfy conditions (1), (3), and (4) of $\S 2$. This follows by straightforward grinding. (b) is obvious. To prove (c) we make use of the following

Lemma. (i) $n x \equiv g \bmod R$ has no solution.

(ii) If $(u, h) \in K$ and $u g+n h \in R$, then $u=0$.

For suppose that there exists an $h \in G$ such that $n h=g+k$ where $k \in R$. Then $g=n\left(h-k /\left[L^{(n-1) / n}+\cdots+L^{1 / n}+1\right]\right)$, a contradiction. Next assume that $(u, h) \in K, u \neq 0$, and $u g+n h \in R$. Then the greatest common divisor of $u$ and $n$ is one. Hence $1=a u+b n$ for $a, b \in I$. $g=a u g+b n g$ and $u g=n(-h)+k$ where $k \in R$. Thus $g=a[n(-h)+k]$ $+b n g \equiv n[a(-h)+b g] \bmod R$, but this contradicts (i). 
We define $(u, h) \in K$ positive if $u g+n h>0$ in $G$. Clearly $(0,0)$ is not positive. Let $(u, h)$ and $(v, k)$ be nonzero elements of $K$.

I. Either $(u, h)$ or $-(u, h)$ is positive. For suppose that $(u, h)$ is not positive, then $u g+n h<0$. If $u=0$, then $-(u, h)=(0,-h)$ and $0 g+n(-h)=-n h>0$. If $u \neq 0$, then by (ii) every element of $-(u g$ $+n h)+R$ is positive. $-(u, h)=(n-u,-h r(n-u)-g)$. $(n-u) g$ $+n(-h r(n-u)-g) \equiv-(u g+n h) \bmod R$. In either case $-(u, h)$ is positive.

II. If $(u, h)$ and $(v, k)$ are positive, then $(u, h)+(v, k)=([u+v] \pi$, $f(u, v)+h r(v)+k)$ is positive. This is obvious if $u=v=0$. Suppose that $u \neq 0$ or $v \neq 0$. If $u+v<n$, then $(u, h)+(v, k)=(u+v, k r(v)+k)$ and $(u+v) g+n(h r(v)+k) \equiv u g+n h+v g+n k \bmod R$. If $u+v=a+n$, then $(u, h)+(v, k)=(a, g+h r(v)+k)$ and $a g+n(g+h r(v)+k) \equiv a g+n g$ $+n h+n k \equiv u g+n h+v g+n k \bmod R$. If $u \neq 0$, then $u g+n h \notin R$ and if $v \neq 0$, then $v g+n k \notin R$. Hence in either case $(u, h)+(v, k)$ is positive.

III. If $(u, h)$ is positive, then $A=(v, k)+(u, h)-(v, k)$ is positive. Suppose that $v=0$. Then $A=(u, k r(u)+h-k)$. If $u=0$, then $A=(0$, $k+h-k)$ and $0 g+n(k+h-k)=k+n h-k>0$. If $u \neq 0$, then $u g+n h$ mod $R$ contains only positive elements, and $u g+n(k r(u)+h-k)$ $\equiv u g+n h \bmod R$. Next suppose that $v \neq 0$. Then $A=(u, f([v+u] \pi$, $n-v)+f(v, u) r(n-v)+k r(u) r(n-v)+h r(n-v)-k r(n-v)-g)$. If $u=0$, then $A=(0, g+k r(n-v)+h r(n-v)-k r(n-v)-g)$ which is positive. Assume $u \neq 0$. If $v+u<n$, then $f([v+u] \pi, n-v)=f(v+u$, $n-v)=g$ and $f(v, u)=0$. Hence $A=(u, g+k r(u) r(n-v)+h r(n-v)$ $-k r(n-v)-g)=(u, b)$, and $u g+n b \equiv u g+n h \bmod R$. If $v+u=a+n$, then $[v+u] \pi+n-v=u$. Hence $A=(u, \quad g r(n-v)+k r(u) r(n-v)$ $+h r(n-v)-k r(n-v)-g)=(u, c)$, and $u g+n c \equiv u g+n h \bmod R$. Therefore $K$ is an $o$-group. Since $n(u, h) \in G^{*}$ and $h>0$ in $G$ if and only if $(0, h)$ is positive in $K$, we have that $K$ is a proper $a$-extension of $G^{*}$. Thus modulo a considerable amount of computation we have a proof of Theorem 3.5.

Remark. This theorem can be sharpened. Suppose that $G$ is an $o$-group of rank 2. Then there exists a $d$-closed $a$-extension $H$ of $G$ such that $H^{1} \cong R$ and $H / H^{1}$ is the $d$-closure of $G / G^{1}$. Moreover if $K$ is another $o$-group that satisfies all these conditions, then $H$ and $K$ are equivalent extensions of $G$. This follows from Theorem 3.3 and the uniqueness of the method of adding a solution of $n x=g$ that we used in the above proof.

4. Construction of $o$-groups. Let $G$ be of rank 2. Then without loss of generality $G=N^{\prime} \times N$ where $N^{\prime}$ and $N$ are subgroups of $R$ and $G$ is a normal $o$-extension of $\bar{N}=0 \times N$ by $N^{\prime}$.

I. $G$ is a splitting extension of $\bar{N}$ by $N^{\prime}$. Then $G$ is completely 
determined by a homormophism of $N^{\prime}$ into the subgroup $L=\{x \in P$ $\mid x N=N\}$ of $P$. Two such extensions (determined by the homomorphisms $r_{1}$ and $r_{2}$ ) are equivalent if and only if there exists $p \in P$ such that $p N^{\prime}=N^{\prime}$ and $r_{1}\left(a^{\prime}\right)=r_{2}\left(p a^{\prime}\right)$ for all $a^{\prime} \in N^{\prime}$. Any homomorphism $r$ of $N^{\prime}$ in to $P$ can be extended to a homomorphism $s$ of $R$ into $P$. For let $Q$ be the $d$-closure of $N^{\prime}$ in $R$. Then for every $q \in Q$ there exists $n \in I$ such that $n q \in N^{\prime}$. Define $v(q)=(r(n q))^{1 / n}$. Then $v$ is the homomorphism of $Q$ into $P$ such that $v\left(a^{\prime}\right)=r\left(a^{\prime}\right)$ for all $a^{\prime} \in N^{\prime} . R=Q \oplus D$. Hence every $x \in R$ has a unique representation $x=q+d$. Define $s(x)=v(q)$. Then $s$ is the desired extension of $r$. Thus by Theorem 3.3 we have: $G$ can be extended to a splitting o-extension of $\bar{R}=0 \times R$ by $R$.

Since $R$ is the direct sum of groups $D a_{\gamma}$ where $D$ denotes the rational numbers and $a_{\gamma} \in R$, a homomorphism $\sigma$ of $R$ into $P$ is determined by its behavior on the $D a_{\gamma}$. But $a_{\gamma} \sigma=p_{\gamma} \in P$, and $d a_{\gamma} \sigma=\left(p_{\gamma}\right)^{d}$ for all $d \in D$.

There are three nonequivalent splitting $o$-extensions of $\bar{R}$ by $R$ for which the defining homomorphism is continuous. For a continuous homomorphism $s$ of $R$ into $P$ is of the form $s(x)=2^{k x}$ for all $x \in R$, where $k$ is a fixed element of $R$. If $k=0$, then $G=R \oplus R$. If $k \neq 0$, then we have the real (one-dimensional) affine group. If $G_{1}$ and $G_{2}$ are determined by $k_{1} \neq 0$ and $k_{2} \neq 0$ respectively, and $k_{1} k_{2}>0$, then the mapping $\pi$ of $\left(a^{\prime}, b\right)$ onto $\left(\left[k_{1} / k_{2}\right] a^{\prime}, b\right)$ is an $o$-isomorphism of $G_{1}$ onto $G_{2}$ such that $x \pi=x$ for all $x \in \bar{R}$.

II. $\bar{N}$ is in the center $Z$ of $G$. ExAmple. Let $\pi$ and $\sigma$ be homomorphisms of $N^{\prime}$ into $N$ such that $\pi\left(a^{\prime}\right) \sigma\left(b^{\prime}\right) \in N$ for all $a^{\prime}, b^{\prime} \in N^{\prime}$. Define $f\left(a^{\prime}, b^{\prime}\right)=\pi\left(a^{\prime}\right) \sigma\left(b^{\prime}\right)$.

III. $G$ is not a splitting extension and $\bar{N}$ is not in the center of $G$. Example. Let $N=R$ and $r$ be a homomorphism of $N^{\prime}$ in to $P$. Define

$$
f\left(a^{\prime}, b^{\prime}\right)= \begin{cases}0 & \text { if } \quad a^{\prime}=0 \text { or } b^{\prime}=0, \\ r\left(b^{\prime}\right)+1 & \text { if } \quad a^{\prime} \neq 0 \text { and } a^{\prime}+b^{\prime}=0, \\ r\left(b^{\prime}\right) & \text { if } \quad a^{\prime} \neq 0, b^{\prime} \neq 0 \text { and } a^{\prime}+b^{\prime} \neq 0 .\end{cases}
$$

Since the above constructions depend only on the existence of a homomorphism of $N^{\prime}$ into $R$ or $P$, the following lemma enables us to extend the construction to 0 -groups $G$ such that $N^{\prime}$ is not a subgroup of $R$.

LEMma. Each of the following properties implies the existence of a nontrivial homomorphism of $G$ onto $R$. (1) $G$ covers a convex subgroup. (2) The rank of $G$ is inversely well ordered. (3) $G$ is abelian and d-closed. (4) There exists a $\gamma \in \Gamma$ such that $G_{\gamma}$ is normal in $G, G^{\gamma} \neq G$, and $G^{\gamma} / G_{\gamma}$ is not in the center of $G / G_{\gamma}$. (5) There exists a $\gamma \in \Gamma$ such that 
$G_{\gamma}$ is normal in $G, G^{\gamma} / G_{\gamma}$ is in the center of $G / G_{\gamma}$, and $G / G_{\gamma}$ splits over a nonzero subgroup $B$ of $G^{r} / G_{\gamma}$.

Proof. If $G$ covers a convex subgroup $C$, then let $\pi=\pi_{1} \pi_{2}$ where $\pi_{1}$ is the natural $o$-homomorphism of $G$ onto $G / C$ and $\pi_{2}$ is an $o$-isomorphism of $G / C$ into $R$. If the rank of $G$ is inversely well ordered, then $G$ covers a convex subgroup. If $G$ is abelian and $d$-closed, then $G / G_{\gamma} \cong G / G^{\gamma} \oplus G^{\gamma} / G_{\gamma}$ for every $\gamma \in \Gamma$. Let $\pi=\phi_{\gamma} \pi_{3} \pi_{\gamma}$ where $\phi_{\gamma}$ is the natural $o$-homomorphism of $G$ onto $G / G_{\gamma}, \pi_{3}$ is the projection of $G / G_{\gamma}$ onto $G^{\gamma} / G_{\gamma}$, and $\pi_{\gamma}$ is an $o$-isomorphism of $G^{\gamma} / G_{\gamma}$ into $R$. Next assume that $\gamma \in \Gamma$ satisfies (4). Then $G / G_{\gamma} \cong\left\{\left(a^{\prime}, a\right) \mid a^{\prime} \in G / G^{\gamma}\right.$ and $\left.a \in G^{\gamma} / G_{\gamma}\right\}$ and $\left(a^{\prime}, a\right)+\left(b^{\prime}, b\right)=\left(a^{\prime}+b^{\prime}, f\left(a^{\prime}, b^{\prime}\right)+a r\left(b^{\prime}\right)+b\right)$ where $r$ is a nontrivial homomorphism of $G / G^{r}$ into the group $A$ of $o$-automorphisms of $G^{\gamma} / G_{\gamma}$. Let $\pi=\pi_{5} r \pi_{6} \pi_{7}$ where $\pi_{5}$ is the natural $o$-homomorphism of $G$ onto $G / G^{\gamma}, \pi_{6}$ is an isomorphism of $A$ into $P$, and $\pi_{7}$ is an isomorphism of $P$ onto $R$. Finally suppose that $\gamma \in \Gamma$ satisfies (5). Then $G / G_{\gamma}=B \oplus D$. Let $\pi=\phi_{\gamma} \pi_{8} \pi_{\gamma}$ where $\pi_{8}$ is a projection of $G / G_{\gamma}$ on to $B$.

REMARK. The above proof indicates (and it is easy to prove) that there exists a nontrivial $o$-homomorphism of $G$ into $R$ if and only if $G$ covers a convex subgroup.

The first two of the following examples have been used in the previous discussion.

4.1. An abelian o-group with all components o-isomorphic to $R$ that is not d-closed. Let $A$ be the large direct sum of the groups $R_{i}$ where $R_{i}=R$ for $i=-1,-2,-3, \cdots$. Define $a=\left(\cdots, a_{i}, \cdots\right)$ positive if $a$ is not the identity and the nonzero component with largest subscript is positive. Then $A$ is an abelian $o$-group (with rank $\omega^{*}$ ), and every component is $o$-isomorphic to $R$. In fact $A$ is $d$-closed. Let $B$ be the subgroup of $A$ generated by the small direct sum of the $R_{i}$ and the element $e \in A$ all of whose components are 1 . Then $B$ is not $d$-closed. For $2 x=e$ has no solution in $B$.

4.2. An o-group with no $d$-closed a-extension. Let $K=$ integers (with their natural order). Let $\Gamma=K \cup \infty$ where $k<\infty$ for all $k \in K$. Let $A$ be the small direct sum of the groups $R_{i}$ where $R_{i}=R$ for all $i \in K$. Then $A$ is an abelian 0 -group (where the order is defined as in Example 4.1). For $a=\left(\cdots, a_{i}, \cdots\right) \in A$ we define $a \sigma=\left(\cdots, b_{i}, \cdots\right)$ where $b_{i}=a_{i-1}$ for all $i \in K$. Clearly $\sigma$ is an $o$-automorphism of $A$. Let $C$ be the cyclic subgroup of the group of all $o$-automorphisms of $A$ that is generated by $\sigma$. Then the mapping of $k \in K$ onto $\sigma^{k}$ is an isomorphism of the group $(K,+)$ onto $C$. Finally let $G=K \times A$, and define $(i, a)+(j, b)=\left(i+j, a \sigma^{j}+b\right)$. Then (with the usual ordering) $G$ is an o-group. In fact $G$ is a splitting extension of $A$ by $K .(0, a)$ 
$\cdot \pi[(1,0)]=(0, a \sigma)$ where $\pi[(1,0)]$ is an inner automorphism. Hence $\pi[(1,0)]$ induces the $o$-permutation $k \rightarrow k+1$ of $K$. Now assume that there exists an $a$-extension $H$ of $G$ that is $d$-closed. Then $H$ contains an element $h$ such that $2 h=(1,0)$. Then $\pi(h)$ must induce an $o$-permutation $\tau$ of $K$ such that $k \tau^{2}=k+1$. But this is impossible.

REMARK. By similar reasoning it can be shown that no $a$-extension of $G$ has its last component $d$-closed.

4.3. An abelian o-group is $d$-closed and has every component o-isomorphic to $R$, but is not a-closed. Let $\Gamma$ have the order type of the reals and let $R_{\gamma}=R$ for every $\gamma \in \Gamma$. Then the small direct sum of the $R_{\gamma}$ satisfies the above conditions.

Finally we consider the following four properties of an o-group $G$ : (A) $G$ is $a$-closed. (B) Every component of $G$ is $d$-closed. (C) Every component of $G$ is $o$-isomorphic with $R$. (D) $G$ is $d$-closed. By the previous theorems, examples, remarks, and the results stated in the introduction the 28 logical relationships between these properties are accounted for except whether or not (A), (B), and (C) imply (D).

\section{BIBLIOGRAPHY}

1. P. Conrad, Embedding theorems for abelian groups with valuations, Amer. J. Math. vol. 75 (1953) pp. 1-29.

2. - On ordered division rings, Proc. Amer. Math. Soc. vol. 5 (1954) pp. 323328.

3. L. Fuchs, The extension of partially ordered groups, Acta Math. Acad. Sci. Hungary vol. 1 (1950) pp. 118-124.

4. P. Jaffard, Theorie arithmetique des anneaux du type de Dedekind II, Bull. Soc. Math. France vol. 81 (1953) pp. 41-61.

5. F. W. Levi, Ordered groups, Proceedings of the Indian Academy of Sciences vol. 16 (1942) pp. 256-263.

6. B. H. Neumann, Adjunction of elements to groups, J. London Math. Soc. vol. 18 (1943) pp. 4-11.

7. O. Schreier, Über die Erweiterung von Gruppen, Teil I, Monatshefte für Mathematik und Physics vol. 34 (1926) pp. 165-180.

The Tulane University of Louisiana 